\title{
A shell model for resin flow and preform deformation in thin-walled composite manufacturing processes
}

\author{
$\mathrm{Da} \mathrm{Wu}^{1} \cdot$ Ragnar Larsson ${ }^{1}$ (D)
}

Received: 11 June 2019 / Accepted: 10 October 2019 / Published online: 17 December 2019

(C) The Author(s) 2020

\begin{abstract}
The paper proposes a novel approach to model the in-plane resin flow in deformable thin-walled fiber preforms for liquid composite molding processes. By ignoring the through-thickness flow in large scale thin-walled components, the 3-D resin flow is simplified to an in-plane flow inside the preform by a specialized divergence theorem. Shell kinematics are used to describe the fiber preform deformation, and the compressible flow is modeled in the context of the free surface flow in porous media. For simplicity and efficiency, the normal stretch, which is driven by the internal fluid and applied external pressure, represents the fiber preform expansion and compression. As compared with full 3-D models, the proposed shell model significantly reduces the problem size, while it still represents the primary physical phenomena during the process. The effects of neglecting the through-thickness flow are illustrated in a numerical example that compares the flow for a set of preforms with different thickness. The model is demonstrated from the numerical example of the mold filling in a doubly curved thin-walled fiber preform. Due to the applied vacuum and the consequent resin flow motion, the relevant deformation of the preform is observed.
\end{abstract}

Keywords Fiber preform deformation $\cdot$ Resin flow $\cdot$ Liquid composite molding $\cdot$ Process modeling $\cdot$ Porous media theory

\section{Introduction}

The class of liquid composite molding (LCM) processes has been widely employed for manufacturing fiber reinforced polymer composite materials (FRPCMs) and helps manufacturers to carve out a niche amid the keen market competition. Since the mid-1980s, the automotive industries started to utilize the resin transfer molding (RTM) method to produce high volume production net shape structural components. Then the vacuum assisted resin transfer molding (VARTM) process sprung up in marine, energy and aerospace industries. The VARTM process can reduce the emission of volatile organic compounds, and produce high-quality FRPCM parts with flexible, handy and lowcost tooling. However, the challenges of defects also appear

Ragnar Larsson
ragnar.larsson@chalmers.se

1 Division of Material and Computational Mechanics, Department of Industrial and Materials Science, Chalmers University of Technology, SE-412 96, Göteborg, Sweden during LCM processes, e.g., dry spots, spring-in, microvoids and thickness variations [1]. The resin flow distribution arrangement dramatically influences the whole filling process, so it is keen to model and simulate the process numerically instead of relying on trial and error physically.

Between the late 80s and early 90s, Chan and Hwang [2] started to solve the pressure distribution in the RTM process based on the Darcy's law, which describes the fluid transfer in porous media. Fracchia et al. [3] employed the control volume finite element method (CVFEM) together with the concept of volume of fraction (VOF) to simulate the mold filling in the RTM process using conforming finite elements. Since then, many results following this approach have been published, e.g., [4, 5]. The modified CVFEM, e.g., [6-8], have been proposed. The boundary element method (BEM), e.g., Um and Lee [9], the level set method, e.g., Soukane and Trochu [10] or Gantois et al. [11] and the smoothed particle hydrodynamics (SPH) method, e.g., [12, 13] are also among the methods of LCM process modeling. What's more, Remacle et al. [14] presented a high-order model using the discontinuous Galerkin method (DGM). $\mathrm{Wu}$ and Larsson [15] proposed a homogenized flow model based on the theory of porous media (TPM) to simulate the 
wet-out RTM process. Another interesting study has been done recently by Dammann and Mahnken [16] who used the phase-field models to model the RTM process.

The research is not just limited to simulate the mold filling flow, but it is also extended to model the fiber preform response coupled with the resin flow. Niaki et al. [17] developed a three-phase integrated flow-stress model. Li and Tucker [18] reported a method to model the consolidation problem. Besides, Wysocki et al. [19-21] have modeled the coupled resin flow/perform deformation problems. As an extension, Rouhi et al. [22-25] presented a series of works based on the continuum mechanics framework.

In contrast to the rigid upper mold used in RTM processes, a plastic membrane is placed on top of the preform in the VARTM process. Thus, the preform deforms due to the difference between the internal and external pressure, which leads to thickness variations in final products. Due to this complexity, the VARTM process is more complicated to model than the RTM process. To simulate the VARTM process, Blais et al. [26] modeled the process as Stokes-Darcy coupled problem. Andriamananjara et al. [27] considered the effect of capillary pressure in their model. Besides, various studies on both resin flow and preform response were reported by researches e.g., [28-33]. However, to solve the full 3-D fluid and solid problems, it demands enormous computational efforts. What's more, it is also a challenge to mesh thin parts and keep the elemental skewness, aspect ratio and warpage in proper quality. On the other hand, the process optimization is an interesting topic for discussion. Hsiao et al. [34] built an optimization framework of flow distribution arrangement by using genetic algorithms. From the industry point of view, the speed of the optimization is vital, which relies on the computational efficiency of the process model.

To simplify the LCM process model for thin-walled FRPCMs, it is assumed that the resin flow is confined to the in-plane of the preform, and that the preform deforms solely along the normal of the mold. Consequently, the preform deformation is represented by a normal stretch variable. Based on the packing law $[35,36]$, an explicit solution of the stretch is derived in terms of the fluid and atmosphere pressure. As a result, the LCM process is simplified to the 2-D in-plane resin flow in the 3D deformable preform. Also, in-plane elements are used based on shell kinematics. The resulting shell model for resin flow and preform deformation in thin-walled process applications significantly reduces the number of degrees of freedom, while the primary physical phenomena of the process can still be represented. The model can be applied at the preliminary process design stage to help industries improving both efficiency and quality of the production. Lastly, the validity of assumptions and the capabilities of the model are illustrated through two numerical examples.

\section{Mass and momentum conservations for non-saturated porous media}

Following the developments in [15], we model the wet-out process of the RTM based on the theory of porous media. A two phase porous media is considered, which consists of the solid fiber preform phase and the homogenized resin/gas fluid phase. Let $n^{s}$ indicate the volume fraction of solid phase, whereas $n^{f}$ represents the volume fraction of the (homogenized) fluid phase. So that $n^{s}$ and $n^{f}$ are interrelated through

$$
\begin{aligned}
& n^{s}+n^{f}=1, \\
& n^{f}=\varphi^{l}+\varphi^{g},
\end{aligned}
$$

where the liquid volume fraction $\varphi^{l}$ and the gas volume fraction $\varphi^{g}$ further subdivide the fluid volume fraction $n^{f}$. We introduce the saturation degree, $0 \leq \xi \leq 1$, to indicate the case of partial saturation. We thus express the liquid and gas volume fraction in Eq. $1 \mathrm{~b}$ in terms of $n^{f}$ and $\xi$ as

$$
\begin{aligned}
& \varphi^{l}=\xi n^{f}, \\
& \varphi^{g}=(1-\xi) n^{f} .
\end{aligned}
$$

The dry $\left(n^{f}=\varphi^{g}\right)$ and full-saturated $\left(n^{f}=\varphi^{l}\right)$ cases are represented as $\xi \rightarrow 0$ and $\xi \rightarrow 1$, respectively. As shown in Fig. 1, the dry and full-saturated parts are separated by a thin boundary, which indicates the flow front position where the gradient of saturation degree exists.

Using Fig. 1, let us introduce the deformation mapping $\boldsymbol{x}=\boldsymbol{\varphi}[X]$ for the solid particles and $\boldsymbol{x}=\boldsymbol{\varphi}[X]=\boldsymbol{\varphi}^{f}\left[X^{f}\right]$ for the fluid particles. The solid phase velocity and the homogenized fluid velocity are then obtained by virtue of the material time derivatives

$$
\begin{gathered}
\boldsymbol{v}=\frac{D^{s} \boldsymbol{\varphi}[\boldsymbol{X}]}{D t}=\dot{\boldsymbol{\varphi}}[\boldsymbol{X}], \\
\boldsymbol{v}^{f}=\frac{D^{f} \boldsymbol{\varphi}^{f}\left[\boldsymbol{X}^{f}\right]}{D t},
\end{gathered}
$$

where $D^{s} \bullet / D t=\bullet$ and $D^{f} \bullet / D t$ denote material time derivatives related to the solid reference configuration $B_{0}$ and fluid reference configuration $B_{0}^{f}$, respectively. Regarding solid particles, we introduce the deformation gradient $\boldsymbol{F}$ and its Jacobian $J$ as

$\boldsymbol{F}=\boldsymbol{\varphi} \otimes \nabla_{X}$ with $J=\operatorname{det}[\boldsymbol{F}]>0$,

where $\nabla_{X}$ is the material gradient operator with respect to the solid phase.

From the principle of mass conservation, we find that the solid contents $M^{s}=J n^{s} \rho^{s}$ and fluid contents $M^{f}=$ $J n^{f} \rho^{f}$ conserve as

$$
\begin{aligned}
& \dot{M}^{s}=0, \\
& \dot{M}^{f}+J \nabla \cdot\left(n^{f} \rho^{f} \boldsymbol{v}^{r f}\right)=0,
\end{aligned}
$$




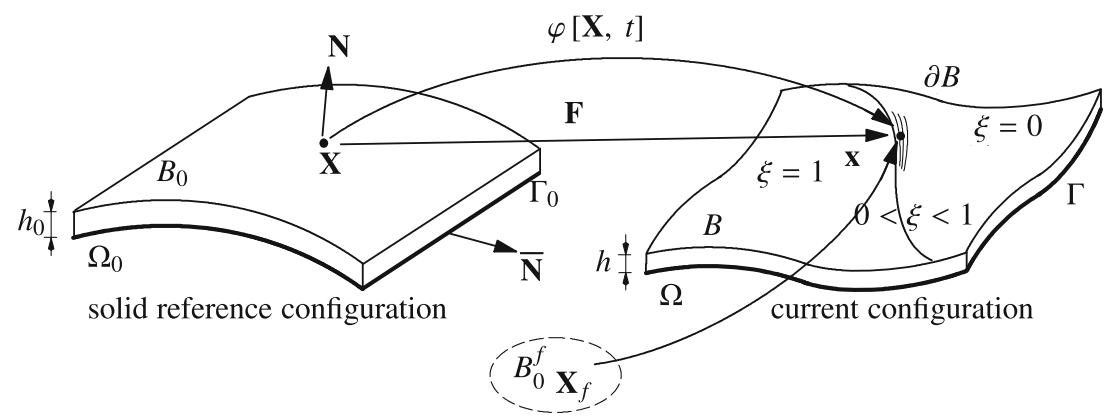

Fig. 1 The reference and current configuration of a partial-saturated deformable thin-walled preform. The thickness of the undeformed thin-walled preform is $h_{0}$ and after deformation the thickness turns

where $\rho^{s}$ and $\rho^{f}$ are the intrinsic densities of the solid and fluid phases, respectively. In particular, the stationarity of $M^{s}$ in Eq. 5a yields $n^{s} \rho^{s} J=n_{0}^{s} \rho_{0}^{s}$, whereby the solid volume fraction is governed by $n^{s}=J^{-1} n_{0}^{s}$, where $\bullet 0$ represents the variable in the reference configuration.

Moreover, based on the arguments of homogenization, see [15], we obtain the homogenized fluid density as

$\rho^{f}=\xi \rho^{l}+(1-\xi) \rho^{g}$,

where $\rho^{l}$ denotes the intrinsic density of the liquid (resin) that is assumed as in-compressible; $\rho^{g}$ is the intrinsic density of the compressible gas. Thus the mixture fluid density is considered as compressible.

Considering the fluid mass flux $\rho^{f} \boldsymbol{v}^{f}$, it can be expressed as a linear combination of the liquid and gas mass fluxes, scaled by the saturation degree,

$\rho^{f} \boldsymbol{v}^{f}=\xi \rho^{l} \boldsymbol{v}^{l}+(1-\xi) \rho^{g} \boldsymbol{v}^{g}$.

Multiplying $\boldsymbol{v}$ on both sides of Eq. 6, we obtain $\rho^{f} \boldsymbol{v}=$ $\xi \rho^{l} \boldsymbol{v}+(1-\xi) \rho^{g} \boldsymbol{v}$; subtracting this by Eq. 7, and introducing the homogenized relative fluid velocity $\boldsymbol{v}^{r f}:=\boldsymbol{v}^{f}-\boldsymbol{v}$, we obtain

$\rho^{f} \boldsymbol{v}^{r f}=\xi \rho^{l} \boldsymbol{v}^{r l}+(1-\xi) \rho^{g} \boldsymbol{v}^{r g}$ with $\boldsymbol{v}^{r l}:=\boldsymbol{v}^{l}-\boldsymbol{v}$ and $\boldsymbol{v}^{r g}:=\boldsymbol{v}^{g}-\boldsymbol{v}$

In view of (2), Eq. 8 can be further elaborated as

$\rho^{f} \boldsymbol{v}^{d f}=\rho^{l} \boldsymbol{v}^{d l}+\rho^{g} \boldsymbol{v}^{d g}$,

where the Darcian velocities $\boldsymbol{v}^{d f}, \boldsymbol{v}^{d l}$ and $\boldsymbol{v}^{d g}$ are defined as

$\boldsymbol{v}^{d f}:=n^{f} \boldsymbol{v}^{r f}, \boldsymbol{v}^{d l}:=\varphi^{l} \boldsymbol{v}^{r l}, \boldsymbol{v}^{d g}:=\varphi^{g} \boldsymbol{v}^{r g}$.

Upon combining the relations (5) and Eq. 6 together with the saturation constraint Eq. 1a, we obtain the total mass conservation of the solid-fluid mixture as

$$
\frac{\dot{J}}{J}+\frac{\rho^{l}-\rho^{g}}{\rho^{f}} n^{f} \dot{\xi}+\frac{n^{f}(1-\xi)}{\rho^{f}} \dot{\rho}^{g}+\frac{1}{\rho^{f}} \nabla \cdot\left(\rho^{f} \boldsymbol{v}^{d f}\right)=0 \text {, }
$$

to $h . B_{0}$ and $B$ denote the region of undeformed and deformed preform respectively, and the preform bottom is named as $\Omega_{0}$ (or $\Omega$ ). In the figure, $N$ is the unit normal of $\Omega_{0}$, whereas $\bar{N}$ is the outward unit normal of the boundary line $\Gamma_{0}$

which is named as pressure equation. Similarly, as to the liquid mass, e.g., $M^{l}=J \varphi^{l} \rho^{l}$, we obtain the balance equation as

$\dot{M}^{l}+J \nabla \cdot\left(\rho^{l} \boldsymbol{v}^{d l}\right)=0$.

Given the fact that the liquid resin phase is incompressible, Eq. 12 can be formulated as an evolution equation of the saturation degree, see also [22] and [15],

$n^{f} \dot{\xi}+\xi \frac{\dot{J}}{J}+\nabla \cdot \boldsymbol{v}^{d l}=0$

Finally, from quasi-static momentum conservation of the mixture porous media, the linear momentum balance yields

$\hat{\boldsymbol{\sigma}} \cdot \nabla+\hat{\rho} \boldsymbol{g}=\mathbf{0} \forall \boldsymbol{x} \in B$,

where $\hat{\rho}=n^{s} \rho^{s}+n^{f} \rho^{f}$, and the total stress $\hat{\sigma}$ relates to the effective (constitutive) stress $\sigma$ of the fiber network and the fluid pressure $p$, viz., Terzaghi effective stress principle,

$\hat{\boldsymbol{\sigma}}=\sigma-p \mathbf{1}$.

From ref. 15, it implies that the deviatoric parts of total stress and effective stress are same, i.e., $\hat{\sigma}_{d e v}=\sigma_{d e v}$, whereby the total pressure $\hat{p}$ is obtained as the summation of the fluid pressure $p$ and the effective pressure $p^{e}$ as

$\hat{p}=p^{e}+p$.

As to the fluid stress response, it is assumed that the fluid is ideal with negligible shear stress. Thereby the intrinsic fluid stress is represented by the fluid pressure $p$, which is homogenized between the pressures of the liquid resin and the gas. It follows from the homogenization in [15], that the (mixture) fluid pressure is an interaction in the degree of saturation $\xi$ between the intrinsic liquid and gas pressures $\left(p^{l}\right.$ and $\left.p^{g}\right)$ written as

$p=\xi p^{l}+(1-\xi) p^{g}$. 


\section{Preform deformation and resin flow analysis}

In this section, we consider the LCM process of a deformable thin-walled preform resting on a fixed lower surface. The preform undergoes the atmospheric pressure $p^{a}$ through a flexible membrane, as shown in Fig. 2a. Given the nature of a thin-walled preform, it is considered as a single director shell surface $\Omega_{0}$ with the unit normal $N$ as indicated in both Figs. 1 and $2 b$, see also [37]. Under the pressure loading in Fig. 2a, we shall assume that the preform can compress or expand only along the normal $N$ via the thickness stretch defined as

$\lambda:=\frac{h}{h_{0}}$,

where $h$ is the current thickness of the preform whereas $h_{0}$ is the undeformed thickness. It is also assumed that the fluid pressure $p$ and the saturation degree $\xi$ keep constant along the thickness direction. We thereby simplify the
3-D Darcy flow to the in-plane flow in an arbitrary curved surface $\Omega$.

\section{Geometry of the thin-walled pressure loaded preform}

In order to define the geometry of the thin-walled pressure loaded preform, its current geometry $B$ is assumed to deform solely with the normal stretch $\lambda$ in Eq. 18 so that $B=B[\lambda]$. The normal stretch field is parameterized in the curve-linear coordinates $\left(\theta^{1}, \theta^{2}, \theta^{3}=\theta\right)$ in Fig $2 \mathrm{~b}$ defined as

$\lambda\left[\boldsymbol{X}_{0}\right]>0 \forall \boldsymbol{X}_{0} \in \Omega_{0}$ with $\Omega_{0}=\left\{\boldsymbol{X}_{0}=\boldsymbol{\Phi}\left[\theta^{1}, \theta^{2}\right],\left[\theta^{1}, \theta^{2}\right] \in \mathcal{A}\right\}$,

where the initial position vector $\boldsymbol{\Phi}\left[\theta^{1}, \theta^{2}\right]$ defines material points on the lower surface $\Omega_{0}$ of the preform. Next, based on the assumption that only normal direction deformation is allowed, the current geometry $B[\lambda]$ is defined in terms of the normal stretch on $\Omega_{0}$ as

$B[\lambda]=\left\{\boldsymbol{x}=\boldsymbol{\Phi}\left[\theta^{1}, \theta^{2}\right]+\theta \lambda\left[\theta^{1}, \theta^{2}\right] \boldsymbol{N}\left[\theta^{1}, \theta^{2}\right],\left[\theta^{1}, \theta^{2}\right] \in \mathcal{A}\right.$ and $\left.\theta \in\left[0, h_{0}\right]\right\}$,

In particular, the initial undeformed geometry $B_{0}=B[1]$ is

obtained as

$B_{0}=\left\{\boldsymbol{X}=\boldsymbol{\Phi}\left[\theta^{1}, \theta^{2}\right]+\theta \boldsymbol{N}\left[\theta^{1}, \theta^{2}\right]\right.$ with $\left[\theta^{1}, \theta^{2}\right] \in \mathcal{A}$ and $\left.\theta \in\left[0, h_{0}\right]\right\}$.

From Eq. 21, we find that

$d \boldsymbol{X}=\boldsymbol{G}_{\alpha}\left[\theta^{1}, \theta^{2}\right] d \theta^{\alpha}+\boldsymbol{N}\left[\theta^{1}, \theta^{2}\right] d \theta=\left(\boldsymbol{G}_{\alpha} \otimes \boldsymbol{G}^{\alpha}+\boldsymbol{N} \otimes \boldsymbol{N}\right) \cdot d \boldsymbol{X}$,

where the co-variant basis vectors (in-plane $\boldsymbol{G}_{\alpha}$ and out-ofplain $\boldsymbol{N}$ ) are defined as

$\boldsymbol{G}_{\alpha}=\frac{\partial \boldsymbol{\Phi}}{\partial \theta^{\alpha}}+\theta \frac{\partial \boldsymbol{N}}{\partial \theta^{\alpha}}=\frac{\partial \boldsymbol{\Phi}}{\partial \theta^{\alpha}}-\theta \boldsymbol{K}_{\alpha \beta} \boldsymbol{G}^{\beta}$,

where $\alpha=1,2$ and $\boldsymbol{G}_{3}=\boldsymbol{G}^{3}=\boldsymbol{N}$, and the curvature of the preform $K_{\alpha \beta}$ is defined as

$K_{\alpha \beta}=\frac{\partial \boldsymbol{G}_{\alpha}}{\partial \theta^{\beta}} \cdot \boldsymbol{N}=-\frac{\partial \boldsymbol{N}}{\partial \theta^{\beta}} \cdot \boldsymbol{G}_{\alpha}$.

Moreover, in Eq. 23 we introduced the contra-variant basis vectors $\boldsymbol{G}^{i}(i=1,2,3)$, which are defined from the identity relationship

$\mathbf{1}=\underbrace{\frac{\partial \boldsymbol{X}}{\partial \theta^{i}}}_{\boldsymbol{G}_{i}} \otimes \underbrace{\frac{\partial \theta^{i}}{\partial \boldsymbol{X}}}_{\boldsymbol{G}^{i}}=\boldsymbol{G}_{i} \otimes \boldsymbol{G}^{i} \rightsquigarrow \boldsymbol{G}^{j}=G^{i j} \boldsymbol{G}_{i}$ with $G^{i j}=\boldsymbol{G}^{i} \cdot \boldsymbol{G}^{j}$, where $G_{i j}$ is the metric tensor. Finally, the infinitesimal area element $\mathrm{d} A$ on the $\Omega_{0}$ and volume element $\mathrm{d} V$ in the $B_{0}$ are formulated in the curve-linear coordinates as

$$
\begin{aligned}
& \mathrm{d} A=\left|\boldsymbol{G}_{1} \times \boldsymbol{G}_{2}\right| d \theta^{1} d \theta^{2}=\sqrt{G} d \theta^{1} d \theta^{2}, \\
& \mathrm{~d} V=\left(\boldsymbol{G}_{1} \times \boldsymbol{G}_{2}\right) \cdot \boldsymbol{N} d \theta^{1} d \theta^{2} d \theta^{3}=\sqrt{G} d \theta^{1} d \theta^{2} d \theta
\end{aligned}
$$

Likewise, from the current geometry in Eq. 20 and the curve-linear coordinates of the preform, we identify the deformation gradient from the linearization

$\mathrm{d} \boldsymbol{x}=\left(\frac{\partial \boldsymbol{\Phi}}{\partial \theta^{\alpha}}+\theta \frac{\partial(\lambda \boldsymbol{N})}{\partial \theta^{\alpha}}\right) d \theta^{\alpha}+\lambda \boldsymbol{N} d \theta=\left(\boldsymbol{g}_{i} \otimes \boldsymbol{G}^{i}\right) \cdot d \boldsymbol{X}=\boldsymbol{F} \cdot d \boldsymbol{X}$,

where the co-variant basis vectors are identified as

$\boldsymbol{g}_{\alpha}=\frac{\partial \boldsymbol{\Phi}}{\partial \theta^{\alpha}}+\theta \frac{\partial(\lambda \boldsymbol{N})}{\partial \theta^{\alpha}}$ with $\alpha=1,2$,

$g_{3}=\lambda N$.

In the following, let us assume that bending effects of the preform can be neglected corresponding to $(\lambda \boldsymbol{N})_{, \alpha} \approx \mathbf{0}$ and $K_{\alpha \beta} \approx \mathbf{0}$, see [37]. Consequently, we can obtain $\boldsymbol{g}_{\alpha}=$ 
a)

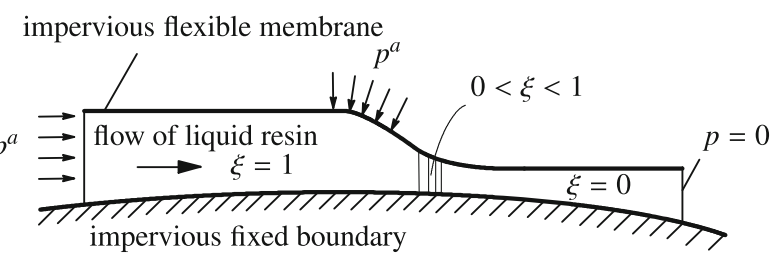

b)

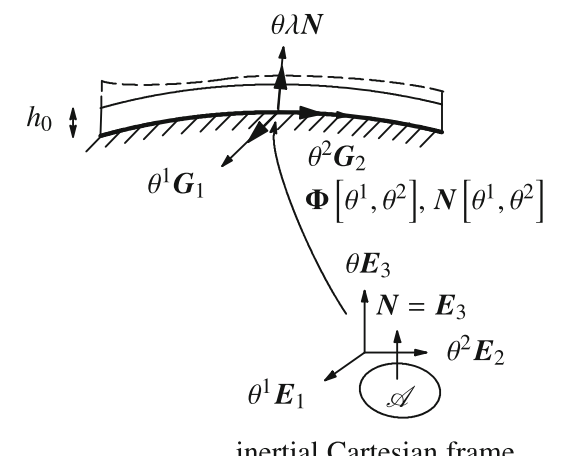

Fig. 2 Considered assumptions of the infusion process of a deformable thin-walled preform: a) a principal sketch of the in-plane flow inside the deformable thin-walled preform; b) the mapping between the undeformed/deformed configuration of the shell model and the inertial Cartesian frame. The figure shows a section of the preform along the $\boldsymbol{G}_{\mathbf{2}}$-basis vector

$\boldsymbol{G}_{\alpha}$, whereby the deformation gradient of the preform is simplified to

$$
\begin{aligned}
\boldsymbol{F}= & \boldsymbol{g}_{i} \otimes \boldsymbol{G}^{i} \\
= & \boldsymbol{G}_{\alpha} \otimes \boldsymbol{G}^{\alpha}+\lambda \boldsymbol{N} \otimes \boldsymbol{N} \\
= & \overline{\mathbf{1}}+\lambda \boldsymbol{N} \otimes \boldsymbol{N} \\
= & \mathbf{1}+(\lambda-1) \boldsymbol{N} \otimes \boldsymbol{N} \\
& \quad \text { with } i=1,2,3 ; \alpha=1,2,
\end{aligned}
$$

where $\overline{\mathbf{1}}$ and $\mathbf{1}=\overline{\mathbf{1}}+\boldsymbol{N} \otimes \boldsymbol{N}$ are the in-plane and 3-D identity tensor respectively. From Eq. 29, it follows that the normal stretch $\lambda$ is the Jacobian of the deformation gradient $\lambda=J=\operatorname{det} \boldsymbol{F}$.

\section{In-plane resin flow in thin-walled preform}

To describe 2-D LCM wet-out process of the thin-walled preform, we consider the fluid resin flow in $B_{0}$ as an inplane flow that is projected to the surface $\Omega_{0}$. To this end, the following form of the divergence theorem is considered for the fluid flow

$\int_{B_{0}} \nabla \cdot \boldsymbol{v} d f \mathrm{~d} V=h_{0} \int_{\Omega_{0}} \bar{\nabla} \cdot \overline{\boldsymbol{v}}^{d f} \mathrm{~d} A=h_{0} \int_{\Gamma_{0}} \overline{\boldsymbol{N}} \cdot \overline{\boldsymbol{v}}^{d f} \mathrm{~d} S$,

where $\bar{\nabla}$ is the in-plane gradient operator, $\bar{N}$ is the outward unit normal of the boundary line $\Gamma_{0}$ on the surface $\Omega_{0}$ (in Fig. 1), and $\overline{\boldsymbol{v}}^{d f}$ is the in-plane fluid Darcy velocity. Please note that the second order tensors and vectors in $\Omega_{0}$ satisfy the orthogonality properties, e.g., $\overline{\boldsymbol{v}}^{d f} \cdot \boldsymbol{N}=0$, and $\overline{\boldsymbol{N}} \cdot \boldsymbol{N}=0$, see also [38]. In view of Eq. 30, we therefore obtain the in-plane pressure gradient $\operatorname{Grad} p$ as

$\operatorname{Grad} p:=\bar{\nabla} p=\frac{\partial p}{\partial \theta^{\alpha}} \frac{\partial \theta^{\alpha}}{\partial \boldsymbol{X}}=p_{, \alpha} \boldsymbol{G}^{\alpha}$.

\section{Constitutive relations}

\section{Fiber packing law}

Considering the mechanical behavior of the deformable fiber preform during the LCM process, a major mechanism is the fiber packing induced by applied pressure. Thus, we consider the Toll's model [35], which is an exponential law in terms of the fiber volume fraction $n^{s}$. In the present model, we limit the analysis to hyper-elastic with the stored free energy defined as

$\psi[J]=\frac{k^{s} E}{m-1}\left(n^{s}\right)^{m}\left(J+m(J-1) J^{m}-J^{m+1}\right)$ with $n^{s}=\frac{n_{0}^{s}}{J}$,

where a slight modification from [35] is made to make sure that $\psi[1]=p^{e}[1]=0$. The $J$ in Eq. 32 denotes the determinant of the deformation gradient, the $k^{s}$ and $m$ are parameters in Toll's packing law, and the variable $E$ represents the elastic Young's modulus of fibers. The effective pressure $p^{e}$ in Eq. 16 is now expressed as

$p^{e}=-\frac{\partial \psi}{\partial J}=k^{s} E\left(\frac{n_{0}^{s}}{J}\right)^{m}\left(1-J^{m}\right)$.

As the fiber volume fraction $n^{s}$ changes, (increasing when $J<1$ and decreasing when $J>1$ ), the packing effect is characterized by the internal contact variations, which is also illustrated in Fig. 3. Hence, given the effective stress

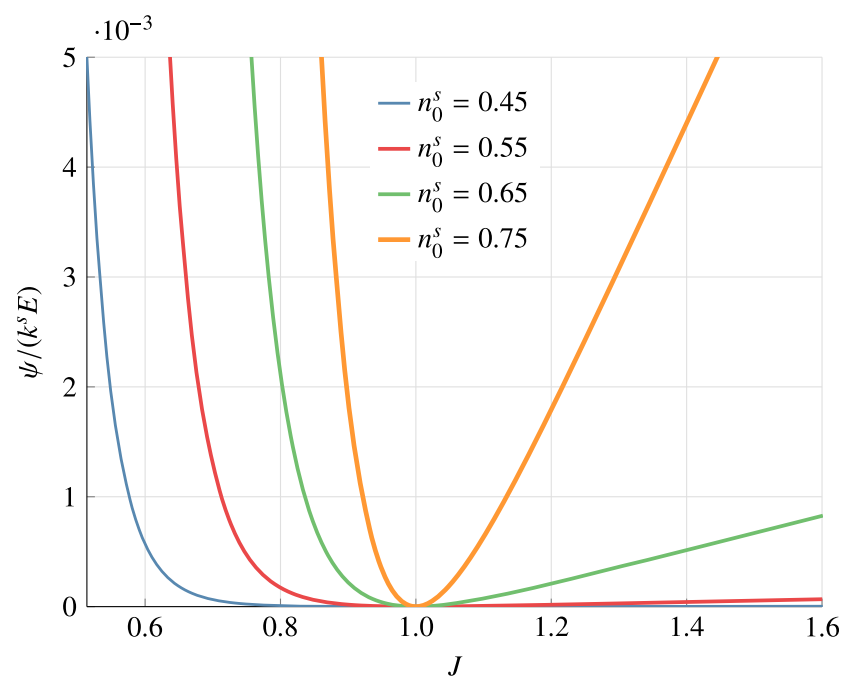

Fig. 3 The normalized fiber preform free energy curves in terms of Jacobian. When the parameter $m$ is set to 15 and various initial fiber volume fractions $n_{0}^{s}$ are chosen, the fiber packing law exhibits that the free energy exponentially increases during compressing, and linearly increases as expanding 
principle in Eq. 16, we obtain the total pressure as $\hat{p}=$ $p^{e}[J]+p$. Moreover, in view of the kinematic assumptions of the preform in Eq. 29, the normal stretch can be expressed as $\lambda=J=\operatorname{det}[\boldsymbol{F}]$.

\section{In-plane Darcy flow}

As to the Darcy flow, we postulate that the 3-D flow is simplified to an effective 2-D flow by ignoring the throughthickness flow, whose transformation is defined by Eq. 30 as

$\overline{\boldsymbol{v}}^{d l}=\varphi^{l} \overline{\boldsymbol{v}}^{r l}=-K^{l} \bar{\nabla} p^{l}$,

$\overline{\boldsymbol{v}}^{d g}=\varphi^{g} \overline{\boldsymbol{v}}^{r g}=-K^{g} \bar{\nabla} p^{g}$,

$K^{\alpha}=\left\{k_{r \alpha} \frac{\hat{\boldsymbol{k}}}{\mu_{\alpha}}\right\}_{\alpha=l, g}$,

where $K^{\alpha}$ is the permeability of the preform relating to the liquid/gas transportation. As suggested by Gebart [39], in turn, $K^{\alpha}$ is defined in terms of the intrinsic permeability $\hat{\boldsymbol{k}}$, the viscosity of the liquid resin/gas $\mu_{\alpha}$ and the relative permeabilitties $k_{r \alpha}$ as according to Burdine [40] are defined as

$k_{r l}=\xi^{3+\frac{2}{n_{b}}}$,

$k_{r g}=(1-\xi)^{2}\left(1-\xi^{1+\frac{2}{n_{b}}}\right)$.

Regarding the intrinsic permeability tensor $\hat{\boldsymbol{k}}$, for simplicity, we consider the isotropic permeability represented by $\hat{\boldsymbol{k}}=\hat{k} \mathbf{1}$. Here, $\hat{k}$ is the deformation dependent isotropic permeability, whose deformation dependence is assumed to follow the Kozeny-Carman equation, see also [39]. Thereby, the permeability is obtained in terms of the fiber volume fraction $n^{s}$ as

$\hat{k}=\frac{r_{s}}{4 C} \frac{\left(1-n^{s}\right)^{3}}{n^{s}}$ with $n^{s}=\frac{n_{0}^{s}}{J}$,

where $r_{s}$ is the particle (or fiber bundle) radius, and $C$ is the Kozeny constant. From that, we can derive the relation between the deformed preform permeability $\hat{k}$ and the undeformed preform permeability $\hat{k}_{0}$ as

$\frac{\hat{k}_{0}}{\hat{k}}=\frac{\left(1-n_{0}^{s}\right)^{3}}{\left(1-\frac{n_{0}^{s}}{J}\right)^{3}} \frac{\frac{n_{0}^{s}}{J}}{n_{0}^{s}}=J^{2}\left(\frac{1-n_{0}^{s}}{J-n_{0}^{s}}\right)^{3}=\frac{1}{J}\left(\frac{n_{0}^{f}}{n^{f}}\right)^{3} \rightsquigarrow \hat{k}=\hat{k}_{0} J\left(\frac{n^{f}}{n_{0}^{f}}\right)^{3}$.

Moreover, by virtue of Eq. 30 and the derivations in [15], the in-plane gradients of the liquid and gas pressure in Eq. 34 are obtained as functions of capillary pressure $p^{c}$, fluid pressure $p$ and saturation degree $\xi$,

$$
\begin{aligned}
& \bar{\nabla} p^{l}=\bar{\nabla} p+\left(1-(1-\xi) \log _{, \xi}\left[p^{c}\right]\right) p^{c} \bar{\nabla} \xi, \\
& \bar{\nabla} p^{g}=\bar{\nabla} p+\left(1+\xi \log _{, \xi}\left[p^{c}\right]\right) p^{c} \bar{\nabla} \xi .
\end{aligned}
$$

We thus conclude that the in-plane Darcy law for the liquid transportation is expressed as

$$
\begin{aligned}
\overline{\boldsymbol{v}}^{d l} & =-K_{p}^{l} \bar{\nabla} p-K_{\xi}^{l} \bar{\nabla} \xi \text { with, } \\
K_{p}^{l} & =K^{l} \text { and }, \\
K_{\xi}^{l} & =K^{l}\left(1-(1-\xi) \log _{, \xi}\left[p^{c}\right]\right) p^{c},
\end{aligned}
$$

and for the gas transportation we obtain

$$
\begin{aligned}
\overline{\boldsymbol{v}}^{d g} & =-K_{p}^{g} \bar{\nabla} p-K_{\xi}^{g} \bar{\nabla} \xi \text { with, } \\
K_{p}^{g} & =K^{g} \text { and }, \\
K_{\xi}^{g} & =K^{g}\left(1+\xi \log _{, \xi}\left[p^{c}\right]\right) p^{c} .
\end{aligned}
$$

Combining Eqs. 10, 39 and 40, we now obtain the homogenized fluid Darcy velocity as

$\overline{\boldsymbol{v}}^{d f}=\frac{\rho^{l}}{\rho^{f}} \overline{\boldsymbol{v}}^{d l}+\frac{\rho^{g}}{\rho^{f}} \overline{\boldsymbol{v}}^{d g}=-K_{p}^{f} \bar{\nabla} p-K_{\xi}^{f} \bar{\nabla} \xi$, where the mixture permeabilities $K_{p}^{f}$ and $K_{\xi}^{f}$ are obtained as

$$
\begin{aligned}
& K_{p}^{f}=\frac{\rho^{l}}{\rho^{f}} K_{p}^{l}+\frac{\rho^{g}}{\rho^{f}} K_{p}^{g}, \\
& K_{\xi}^{f}=\frac{\rho^{l}}{\rho^{f}} K_{\xi}^{l}+\frac{\rho^{g}}{\rho^{f}} K_{\xi}^{g} .
\end{aligned}
$$

\section{Capillary pressure and universal gas law}

The capillary pressure in Eqs. 38 to 40 represents the difference between the gas and liquid pressure, we apply the phenomenological model by Brooks [41]

$p^{c}[\xi]=p^{g}-p^{l}=p^{e n t} \xi^{-\frac{1}{n_{b}}}$,

where $p^{\text {ent }}>0$ denotes the entry pressure and $n_{b}>0$ controls the shape of the capillary curve. By combining Eqs. 43 and 17, the intrinsic pressures of liquid and gas are obtained completely in terms of fluid pressure $p$ and saturation degree $\xi$ as

$$
\begin{aligned}
p^{l} & =p-(1-\xi) p^{c}[\xi] \\
p^{g} & =p+\xi p^{c}[\xi] .
\end{aligned}
$$


As to the compressible gas density $\rho^{g}$, the universal gas law shows

$\rho^{g}=k^{g} p^{g}=k^{g}\left(p+\xi p^{c}[\xi]\right)$ with $k^{g}=\frac{m^{g}}{R T}$,

where $R$ is the universal gas constant and $T$ is the absolute temperature.

\section{Boundary value problems}

To solve the present process model, we introduce the mass balance relations Eqs. 11 and 13 and the quasistatic momentum balance Eq. 14 in weak form. The weak

$\int_{B_{0}} \hat{\boldsymbol{S}}: \delta \boldsymbol{E} \mathrm{d} V=\int_{B_{0}}\left(\boldsymbol{S}-J p \boldsymbol{C}^{-1}\right):\left(\delta \boldsymbol{F}^{t} \cdot \boldsymbol{F}\right) \mathrm{d} V=h_{0} \int_{\Omega_{0}} \boldsymbol{N} \cdot \overline{\boldsymbol{t}}_{1} \delta \lambda \mathrm{d} A \forall \delta \lambda$, forms are formulated in terms of the shell kinematics and the coordinate systems introduced in "Geometry of the thin-walled pressure loaded preform". From "Fiber packing law", we also notice that it suffices to represent deformation gradient $\boldsymbol{F}$ and its Jacobian $J=\operatorname{det}[\boldsymbol{F}]$ by the stretch field $\lambda \in \mathcal{L}_{2}\left[\Omega_{0}\right]$. So the present model aims to solve the fields of fluid pressure $p$, saturation degree $\xi$ and stretch $\lambda$.

\section{Weak form of momentum balance}

The weak form of the momentum balance Eq. 14 is equivalent to the principle of virtual work, which is formulated in the reference configuration $B_{0}$ and in the absence of the gravitational force as where $\overline{\boldsymbol{t}}_{1}$ is the nominal traction vector acting on a surface element $\mathrm{d} A \in \Omega_{0}$. In particular, $\boldsymbol{N} \cdot \overline{\boldsymbol{t}}_{1}:=-p^{a}$ is the prescribed atmospheric pressure at the top surface of the preform, as shown in Fig. 2a. We also introduced the symmetric total second Piola-Kirchhoff stress $\hat{S}$ from the pull-back transformation of the total Cauchy stress $\hat{\boldsymbol{S}}=$ $J \boldsymbol{F}^{-1} \cdot \hat{\boldsymbol{\sigma}} \cdot \boldsymbol{F}^{-t}$. By implementing the Terzaghi stress principle Eq. 15, we obtain

$\hat{\boldsymbol{S}}=J \boldsymbol{F}^{-1} \cdot(\boldsymbol{\sigma}-p \mathbf{1}) \cdot \boldsymbol{F}^{-t}=\boldsymbol{S}-J p \boldsymbol{C}^{-1}$ with $\boldsymbol{S}=J \boldsymbol{F}^{-1} \cdot \boldsymbol{\sigma} \cdot \boldsymbol{F}^{-t}$,

where $\boldsymbol{C}:=\boldsymbol{F}^{t} \cdot \boldsymbol{F}$ is the right Cauchy-Green deformation tensor and $\boldsymbol{S}$ is the effective (constitutive) second PiolaKirchhoff stress.

As mentioned in Eq. 29, the deformation gradient simplifies to

$\boldsymbol{F}=\mathbf{1}+(\lambda-1) \boldsymbol{N} \otimes \boldsymbol{N}$ with $\lambda=J=\operatorname{det}[\boldsymbol{F}]$,

consequently, the packing law Eq. 32 yields the effective stress response as

$\boldsymbol{S}=2 \frac{\partial \psi}{\partial \boldsymbol{C}}=-p^{e} J \boldsymbol{C}^{-1}=-p^{e} \lambda \boldsymbol{C}^{-1}$.

Moreover, we also obtain the explicit expressions for the right Cauchy-Green deformation $\boldsymbol{C}=\boldsymbol{F}^{t} \cdot \boldsymbol{F}$ and its inverse $\boldsymbol{C}^{-1}$ from Eq. 48

$$
\begin{aligned}
& \boldsymbol{C}=\mathbf{1}+\left(\lambda^{2}-1\right) \boldsymbol{N} \otimes \boldsymbol{N}, \\
& \boldsymbol{C}^{-1}=\mathbf{1}+\left(\lambda^{-2}-1\right) \boldsymbol{N} \otimes \boldsymbol{N},
\end{aligned}
$$

where the last equality was obtained using the ShermanMorrison formula.
In view of Eqs. 49 and 50, the virtual work in Eq. 46 is now worked out as

$$
\begin{aligned}
\int_{B_{0}}\left(\delta \boldsymbol{F}^{t} \cdot \boldsymbol{F}\right):\left(\boldsymbol{S}-J p \boldsymbol{C}^{-1}\right) \mathrm{d} V & =\int_{B_{0}}(\boldsymbol{N} \otimes \boldsymbol{N}):\left(\boldsymbol{S}-\lambda p \boldsymbol{C}^{-1}\right) \lambda \delta \lambda \mathrm{d} V \\
& =\int_{B_{0}}\left(\boldsymbol{N} \cdot \boldsymbol{S} \cdot \boldsymbol{N}-\boldsymbol{N} \cdot \boldsymbol{C}^{-1} \cdot \boldsymbol{N} \lambda p\right) \lambda \delta \lambda \mathrm{d} V \\
& =\int_{B_{0}}\left(-p^{e}-p\right) \boldsymbol{N} \cdot \boldsymbol{C}^{-1} \cdot \boldsymbol{N} \lambda^{2} \delta \lambda \mathrm{d} V \\
& =\int_{B_{0}}\left(-p^{e}-p\right) \lambda^{-2} \lambda^{2} \delta \lambda \mathrm{d} V \\
& =-h_{0} \int_{\Omega_{0}}\left(p^{e}+p\right) \delta \lambda \mathrm{d} A \\
& =h_{0} \int_{\Omega_{0}} \boldsymbol{N} \cdot \overline{\boldsymbol{t}}_{1} \delta \lambda \mathrm{d} A \\
& =-h_{0} \int_{\Omega_{0}} p^{a} \delta \lambda \mathrm{d} A
\end{aligned}
$$

Hence, we find that the applied pressure $p^{a}$ balances with the effective and the fluid pressures, $p^{e}$ and $p$. From this balance relationship together with the constitutive relation Eq. 33, relating the effective pressure $p^{e}$ and the stretch $\lambda$, we obtain

$p^{e}[\lambda]=k^{s} E\left(n_{0}^{s}\right)^{m}\left(\lambda^{-m}-1\right)=p^{a}-p$,

whereby the normal stretch field can be resolved explicitly from the hyper-elastic fiber packing law as

$\lambda=\left(1+\frac{p^{a}-p}{k^{s} E\left(n_{0}^{s}\right)^{m}}\right)^{-1 / m}$. 


\section{Weak formulation of mass balance}

Let's consider the in-plane flow in "In-plane Darcy flow" on the surface $\Omega_{0}$ that is bounded by $\Gamma_{0}$. We introduce the test functions for the fields of fluid pressures and saturation degrees as

$$
\begin{aligned}
& P=\left\{\eta[x] \in \mathcal{H}^{1}\left[\Omega_{0}\right] \mid \eta=0 \text { on } \partial_{p} \Omega_{0}\right\}, \\
& S=\left\{\chi[x] \in \mathcal{H}^{1}\left[\Omega_{0}\right] \mid \chi=0 \text { on } \partial_{\xi} \Omega_{0}\right\},
\end{aligned}
$$

where we prescribe the fluid pressure and the saturation degree along the boundaries $\partial_{p} \Omega_{0}$ and $\partial_{\xi} \Omega_{0}$, which satisfy that $\left(\partial_{p} \Omega_{0} \cup \partial_{\xi} \Omega_{0}\right) \subset \Gamma_{0}$. By using the relation $J=\lambda$ in Eqs. 11 and 13, we thus obtain the weak form of the pressure and saturation equation projected onto the surface $\Omega_{0}$ as

$$
\begin{array}{r}
\left.h_{0} \int_{\Omega_{0}}\left(\eta\left(\dot{\lambda} \rho^{f}+\left(\lambda-n_{0}^{s}\right)\right)\left(\left(\rho^{l}-\rho^{g}\right) \dot{\xi}+(1-\xi) \dot{\rho}^{g}\right)\right)-\lambda \rho^{f} \bar{\nabla} \eta \cdot \overline{\boldsymbol{v}}^{d f}\right) \mathrm{d} A \\
=h_{0} \int_{\Gamma_{0}} \eta Q \mathrm{~d} S \quad \forall \eta \in P,
\end{array}
$$

$h_{0} \int_{\Omega_{0}}\left(\chi\left(\left(\lambda-n_{0}^{s}\right) \dot{\xi}+\xi \dot{J}\right)-\lambda \bar{\nabla} \chi \cdot \overline{\boldsymbol{v}}^{d l}\right) \mathrm{d} A=h_{0} \int_{\Gamma_{0}} \chi H \mathrm{~d} S \quad \forall \chi \in S$,

where $Q=\overline{\boldsymbol{N}} \cdot \overline{\boldsymbol{v}}^{d f}, H=\overline{\boldsymbol{N}} \cdot \overline{\boldsymbol{v}}^{d l}$, which are the in-plane fluxes across the boundary $\Gamma_{0}$.

\section{FE interpolations}

The weak forms Eqs. 55 and 56 are solved by the finite element method, which is stabilized by the streamline upwind/Petrov-Galerkin (SUPG) method, as discussed in [15]. The domain $\Omega_{0}$ is discretized by bilinear four-node element. The primary variables $p$ and $\xi$ are interpolated as nodal summation forms,

$$
\begin{aligned}
p \rightsquigarrow p_{h}[\boldsymbol{X}, t] & =\sum_{I=1}^{\mathrm{NNO}} N^{I}[\boldsymbol{X}] p^{I}[t], \eta[\boldsymbol{X}] \rightsquigarrow \eta_{h}[\boldsymbol{X}], \\
\xi \rightsquigarrow \xi_{h}[\boldsymbol{X}, t] & =\sum_{I=1}^{\mathrm{NNO}} N^{I}[\boldsymbol{X}] \xi^{I}[t], \chi[\boldsymbol{X}] \rightsquigarrow \chi_{h}[\boldsymbol{X}],
\end{aligned}
$$

where $\left\{N^{I}\right\}_{I=1, \ldots, \mathrm{NNO}}$ are shape functions, and $\xi^{I}$ and $p^{I}$ are the time-dependent nodal saturation degree and fluid pressure respectively. Furthermore, we obtain the discretized in-plane gradient from Eq. 57 as

$$
\begin{aligned}
\bar{\nabla} \xi \rightsquigarrow \bar{\nabla} \xi_{h} & =\sum_{I=1}^{\mathrm{NNO}} \bar{\nabla} N^{I}[\boldsymbol{X}] \xi^{I}[t], \\
\bar{\nabla} p \rightsquigarrow \bar{\nabla} p_{h} & =\sum_{I=1}^{\mathrm{NNO}} \bar{\nabla} N^{I}[\boldsymbol{X}] p^{I}[t],
\end{aligned}
$$

According to the Eq. 53, we can write out the elemental stretch $\lambda^{e l}$ as

$$
\lambda^{e l}=\left(1+\frac{p^{a}-\sum_{i=1}^{4} N^{i}[x] p^{i}[t]}{k^{s} E\left(n_{0}^{s}\right)^{m}}\right)^{-1 / m} .
$$

Hence, the nodal stretch can be calculated from the average of neighboring elements,

$\lambda^{I}=\frac{1}{\mathrm{NE}} \sum_{I=1}^{\mathrm{NE}} \lambda_{I}^{e l}$

where $N E$ denotes the number of elements that is adjacent to $\lambda^{I}$. Thus, the field $\lambda$ is interpolated as

$\lambda \rightsquigarrow \lambda_{h}[\boldsymbol{X}, t]=\sum_{I=1}^{\mathrm{NNO}} N^{I}[\boldsymbol{X}] \lambda^{I}[t]$.

\section{Numerical examples}

Two representative examples are presented to validate the 2-D assumptions and demonstrate the model. Table 1 lists the parameters used in both examples. At the infusion inlet, the pressure is set to $p_{0}=1$ atm and the corresponding saturation degree is given as $\xi_{0}=1.0$. At the outlet, the boundary conditions are determined by $p_{1}=3.2 \mathrm{mbar}$ and $\partial_{x} \xi=0$; the initial values are set to ${ }^{0} \xi=\xi(\boldsymbol{x}, 0)=$ 
Table 1 Parameters of the fiber preform, resin and environment

\begin{tabular}{llll}
\hline Parameters & Unit & Identification & Value \\
\hline$n_{0}^{s}$ & - & Fiber volume fraction & 0.411 \\
$\hat{k}_{0}$ & {$\left[\mathrm{~m}^{2}\right]$} & Intrinsic permeability & $2.537 \times 10^{-9}$ \\
$\rho^{l}$ & {$\left[\mathrm{~kg} / \mathrm{m}^{3}\right]$} & Resin density & 1010 \\
$\mu_{l}$ & {$[\mathrm{~Pa} \cdot \mathrm{s}]$} & Resin viscosity & 0.1 \\
$\mu_{g}$ & {$[\mathrm{~Pa} \cdot \mathrm{s}]$} & Gas viscosity & $1.983 \times 10^{-5}$ \\
$n_{b}$ & - & Capillary pressure constant & 1.9 \\
$p^{\text {ent }}$ & {$[\mathrm{Pa}]$} & Entry pressure & $0.134 \times 10^{6}$ \\
$m^{g}$ & {$[\mathrm{~kg} / \mathrm{mol}]$} & Gas molar mass & $2.897 \times 10^{-2}$ \\
$R$ & {$[\mathrm{~J} / \mathrm{K} \cdot \mathrm{mol}]$} & Ideal gas constant & 8.314 \\
$T$ & {$[\mathrm{~K}]$} & Absolute temperature & 293 \\
$k^{s}$ & - & Packing law factor & 0.01 \\
$E$ & {$[\mathrm{~Pa}]$} & Young's modulus of fibers & $7.0 \times 10^{10}$ \\
$m$ & - & Packing law exponent & 15 \\
\hline
\end{tabular}

0.001 and ${ }^{0} p=p(\boldsymbol{x}, 0)=3.2$ mbar. Ideally, the initial field ${ }^{0} \xi$ is very close to zero, but numerical singularity problems appear when ${ }^{0} \xi$ is chosen too small. Thus the current value of ${ }^{0} \xi$ has been chosen small enough, while it avoids numerical singularity.

\section{Comparison of the resin flow in thick- and thin-walled fiber preforms}

To verify the in-plane flow assumption in thin-walled preforms, we studied two different models when the preform is considered fixed with $\lambda=1$. The considered preform is a curved tunnel that starts and ends with a 0.2 meters long straight path to steady the flow; there is an 0.8 meters radius curve in between that introduces an unevenly distributed through-thickness flow as shown in Fig. 4. Following [15], a 2-D model is first made for capturing both the in-plane and through-thickness resin flow of the curved preform in Fig. 4a. Second, a 3-D shell model is built for the same geometry; however; because there is no flow along the transverse and through-thickness direction of the preform, the flow can be considered as 1-D in the longitudinal direction, as shown in Fig. 4b. The infusion starts from the inlet and ends at the outlet as depicted in Fig. 4. In order to study the influence of the through the thickness flow as induced by the curved preform, a global saturation degree measure $\tilde{\xi}$ is introduced to represent overall significance of the through the thickness flow effect. The measure $\tilde{\xi}$ is defined as

$\tilde{\xi}=\frac{\sum_{i=1}^{N N O} \xi^{i}}{N N O}$

where the $N N O$ is the number of nodes, and the $\xi^{i}$ denotes the saturation degree at the $i$ th node.

By comparing the $\tilde{\xi}$ in the 2-D plane and shell models, we thus measure the importance of the through-thickness flow for various thickness to length ratios, $t / L$, of the preform. The thickness $t$ varies among $0.05,0.1,0.15$ and 0.2 meters. For each $t / L$ case, the global saturation degree $\tilde{\xi}$ is computed and compared between two models. For the very thick preform $t / L=12 \%$, Fig. 4 a shows the unevenly distributed through-thickness flow for the 2-D plane model, i.e., due to the curved geometry the inner radius flow is faster than the outer radius flow. Figure 5 shows that the percent error, $\delta=\left|\left(\overline{\boldsymbol{\xi}}_{\text {plane }}-\overline{\boldsymbol{\xi}}_{\text {shell }}\right) / \overline{\boldsymbol{\xi}}_{\text {shell }}\right| \times 100 \%$, decreases linearly as the preform thickness decreases. When the ratio $t / L$ is reduced below $6 \%$, the through-thickness flow effect is significantly reduced. For example, a thin preform of $t / L=3 \%$ yields a difference of $1.5 \%$, which justifies the in-plane flow assumption made for the present thin-walled case.
Fig. 4 The saturation degree $\xi$ in $\mathbf{a}$ plane model and $\mathbf{b}$ shell model after 150 seconds of infusion. The mesh size equals to $l_{e}=0.01$ meters for both models and the total infusion length $L \approx 1.65 \mathrm{~m}$

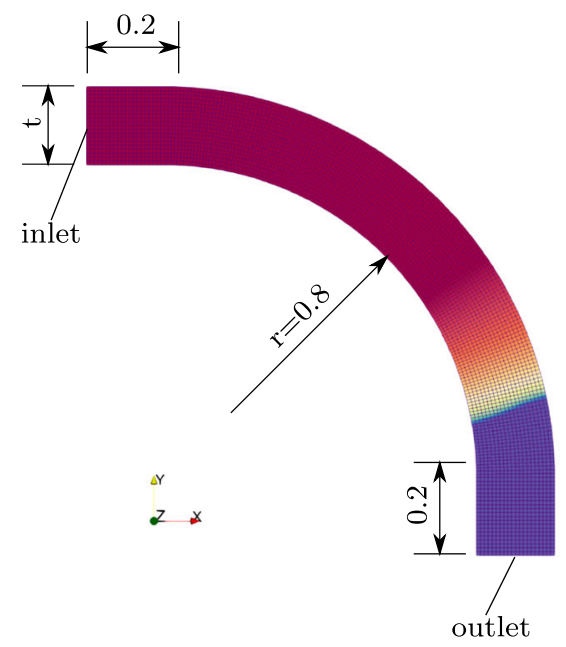

a) plane model $(t=0.2 \mathrm{~m})$

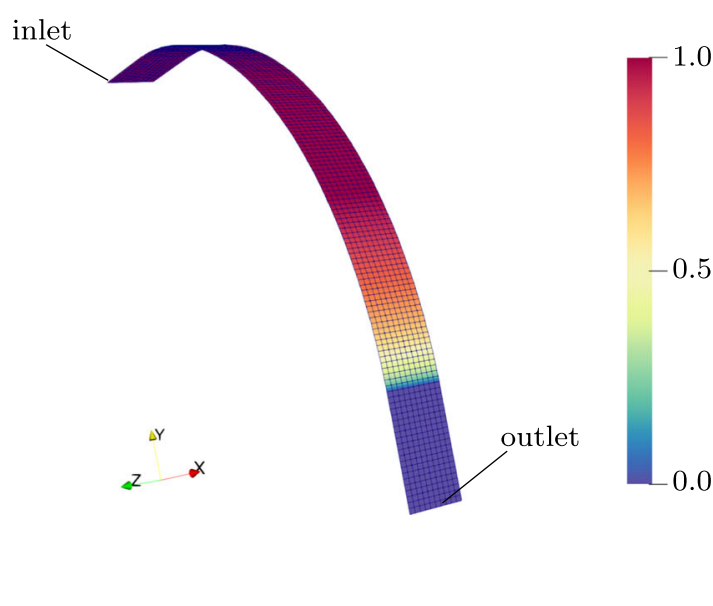

b) shell model 


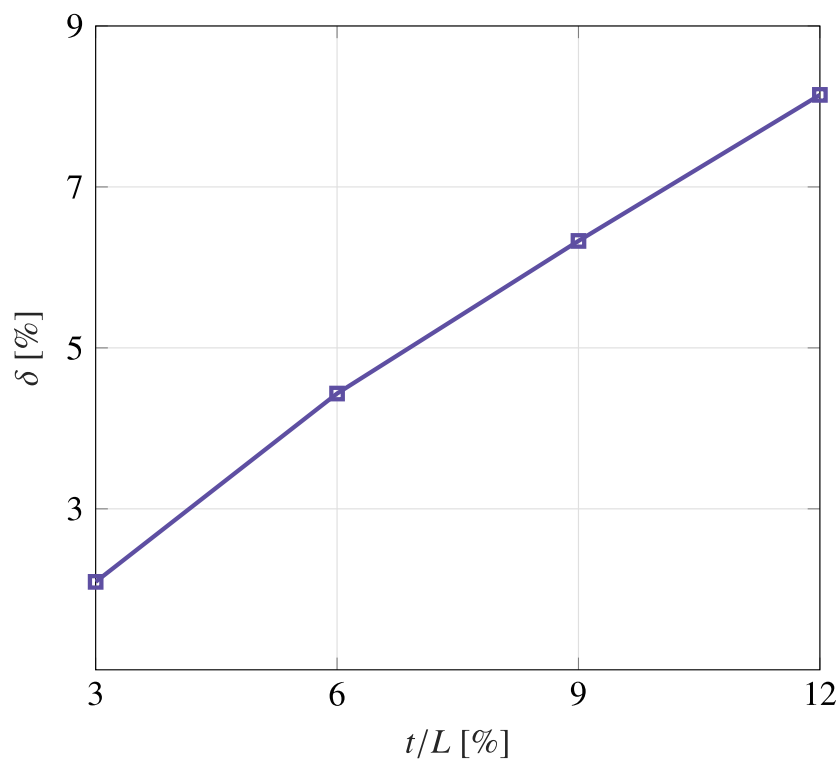

Fig. 5 The differences of the global saturation degree between the plane and shell model after 150 seconds of infusion

\section{LCM process of doubly curved thin-walled preform}

To investigate the capabilities of the proposed model for simulating the infusion of a deformable thin-walled preform, we consider a LCM process example as shown in Fig. 6. The black edges including the hole are impervious, in contrast, the blue edges are the resin inlet and outlet. The curvature $R$ and side length $l$ of the surface $\Omega_{0}$ are 1 meter. The inlet is 0.25 meters wide and locates in the middle of the northwest edge, and the opening has the diameter $d=$ 0.2 meters located at the center.

We choose four paths to represent the results. The first one is the line from the middle of the inlet to the outlet middle, viz., Center; the second path is the "northeast" edge in the Fig. 6, viz., Side; the next one is the line that equally splits the partition between the Center and Side, viz., Middle; and last, the "northwest" edge is named as Front.
Four meshes are employed to discretize the preform, which are the coarse mesh (mesh 1: 163 elements), the regular mesh (mesh 2: 436 elements), the fine mesh (mesh 3: 1067 elements) and the finest mesh (mesh 4: 1882 elements). In addition, four different time step sizes are chosen as, $5 \times 10^{-2}$ (time step 1) $1 \times 10^{-2}$ (time step 2), $1 \times 10^{-3}$ (time step 3 ) and $5 \times 10^{-4}$ (time step 4) seconds.

The mesh size convergence study is based on the difference of the global saturation degree $\tilde{\xi}$ of the coarse, regular and fine meshes relating to the finest mesh. The time step $\Delta t=1 \times 10^{-3}$ seconds is selected and the infusion ends at 150 seconds. Because the number of time steps is fixed, the error with respect to the spatial discretization $e_{m}$ is defined as the root-mean-square error (RMSE) as,

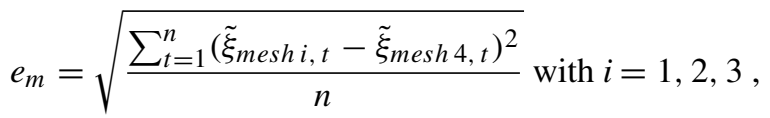

where $n$ is the total number of time steps; $\tilde{\xi}_{\text {mesh } i, t}$ denotes the global saturation degree of the $i$-th mesh scheme at $t$-th second. Figure 7 a shows that the convergence rate decreases as the element number increases. The error dropping from the coarse to the regular mesh is much higher than the dropping from the regular to the fine mesh. Especially, the error reduction from the regular to the fine mesh is limited small. Thus, the fine mesh is "fine enough" for the given case.

Figure $7 \mathrm{~b}$ shows the convergence study of the time step size when the fine mesh is chosen. Since the mesh size is fixed in this study, the error is compared with the nodal saturation degree $\xi$ of the time steps 1,2 and 3 relating to the time step 4 . With respect to the time discretization, the error $e_{t}$ is defined as,

$e_{t}=\sqrt{\frac{\sum_{i=1}^{N N O}\left(\xi_{\text {timestep } n, i}-\xi_{\text {timestep } 4, i}\right)^{2}}{N N O}}$ with $i=1,2,3$,

where $N N O$ equals to the total node numbers of the fine mesh scheme; $\tilde{\xi}_{\text {time step } n, i}$ represents the $i$-th node of $n$-th time step size case. Similar to the convergence study of the
Fig. 6 Infusion example of a 3 -D specimen with dimension and boundary conditions

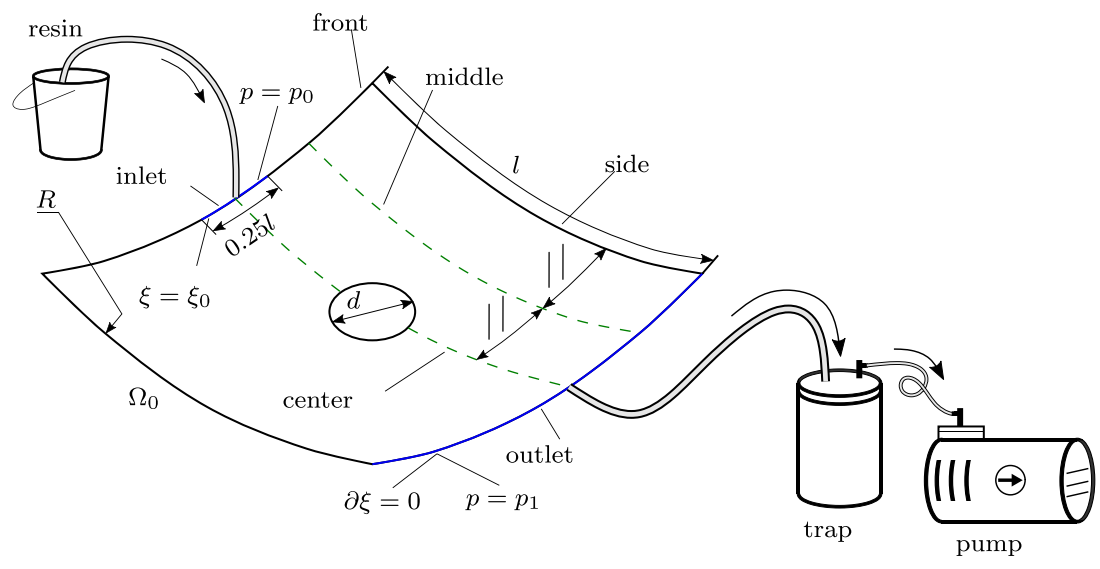


Fig. 7 Convergence studies after 150 seconds of infusion

Fig. 8 Resin flow locations at $12,46,65,137,217$ and 300 seconds after injection started
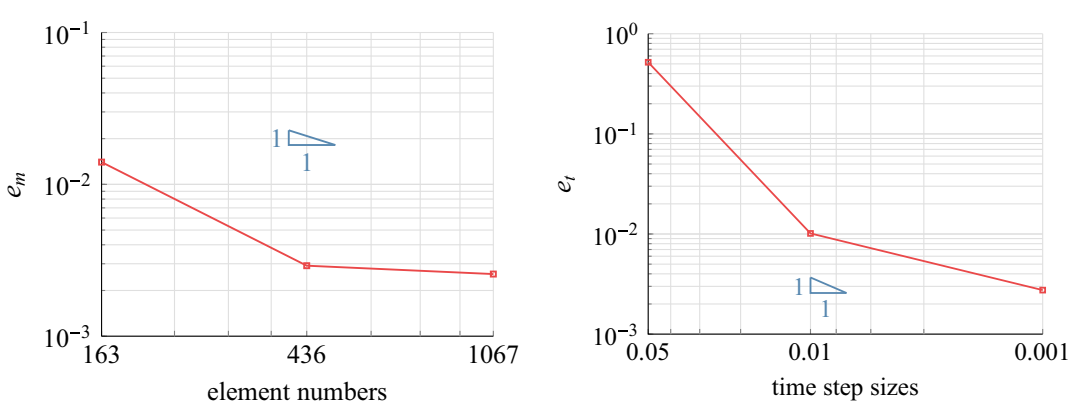

(a) RMSE of global saturation degree of the coarse, (b) RMSE of the saturation degree of the coarse, regregular and fine meshes relating to the finest mesh. ular and fine time step sizes relating to the finest time The time step size is fixed to $1 \times 10^{-3}$ seconds. step size. The fine mesh is used.
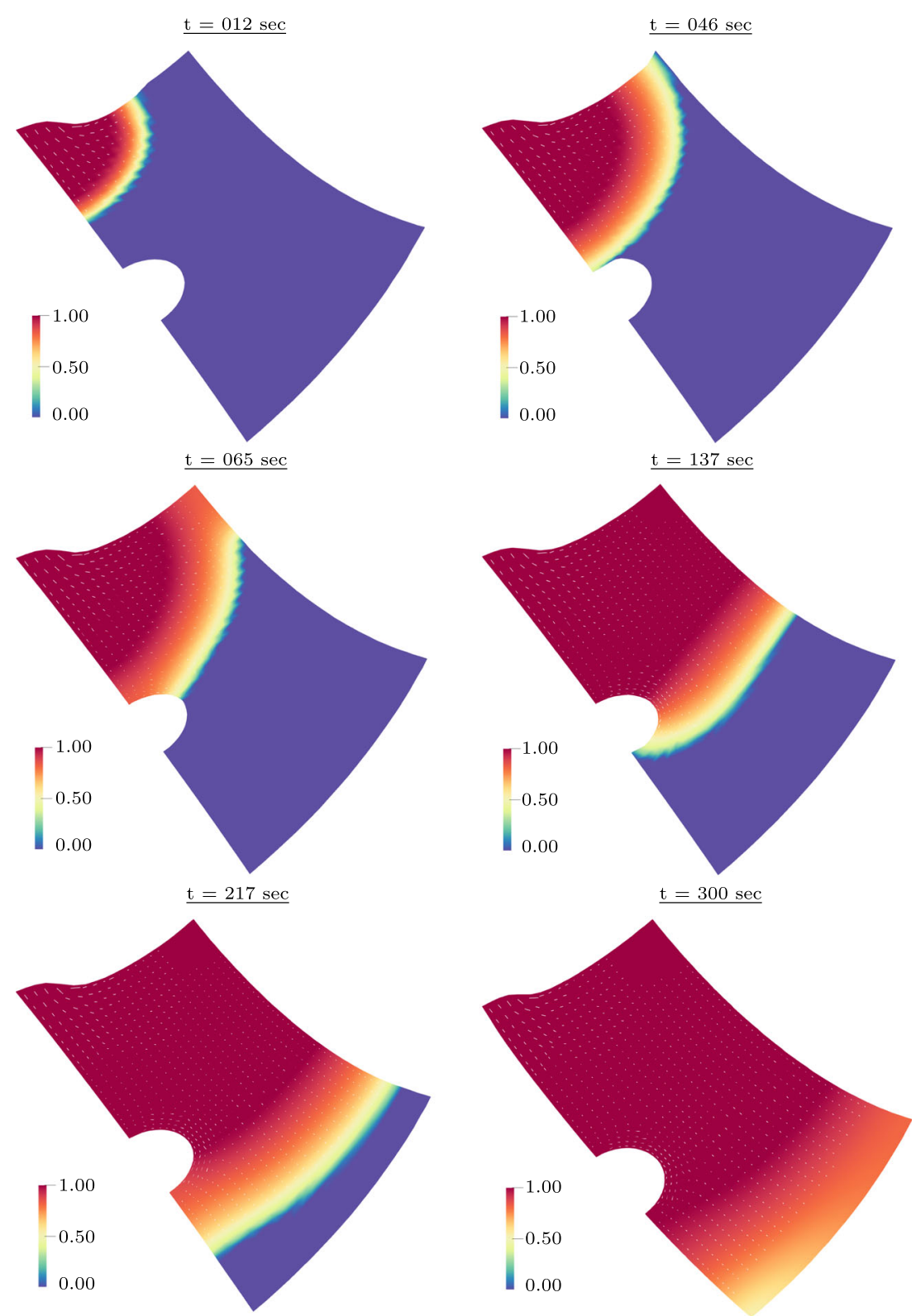
mesh size, the error drops significantly from 0.05 seconds to 0.01 seconds; however, the error decreases much slower when the time step size decreases from 0.01 seconds to 0.001 seconds. Because of this, the 0.001 seconds is chosen as the time step size to save computational effort while still keeping a good accuracy.

It is also interesting to track the flow in the deformable preform. Figure 8 plots the resin flow patterns during the infusion process. The red regions represent the fullsaturated parts of the preform; and the blue regions indicate the dry parts; the gradients between red and blue illustrate the process zone, where the flow front locates. The tiny white lines show the flow directions in each element.

At the beginning of the process $(0-50$ seconds), the resin flow moves towards all directions simultaneously and forms a sector pattern. Once the flow reaches the hole $(50-140$ seconds), the hole drags down the neighboring flow speed. As a result, the side flow gradually moves faster than the center flow. At 137 seconds, the outside flow has already caught up the inner flow around the hole. When the entire flow front has passed the hole, the flow front forms a nearly straight line as shown at 217 seconds.

Figure 9 shows the pressure distribution. We can notice that there are local bands of low-pressure zones (blue bands) around flow fronts. The pressure drops to the bottom where the global minimum pressure locates, then just after the flow front, it rises again and forms a local pressure peak.

We also plot the preform profiles along different paths in Fig. 10, by using the finest mesh and $1 \times 10^{-3}$ seconds as time step size. Due to the vacuum, the preform is
Fig. 9 Pressure distribution at $12,46,65,137,217$ and 300 seconds after injection started. The color bar shows the pressure in the unit of $\mathrm{MPa}$
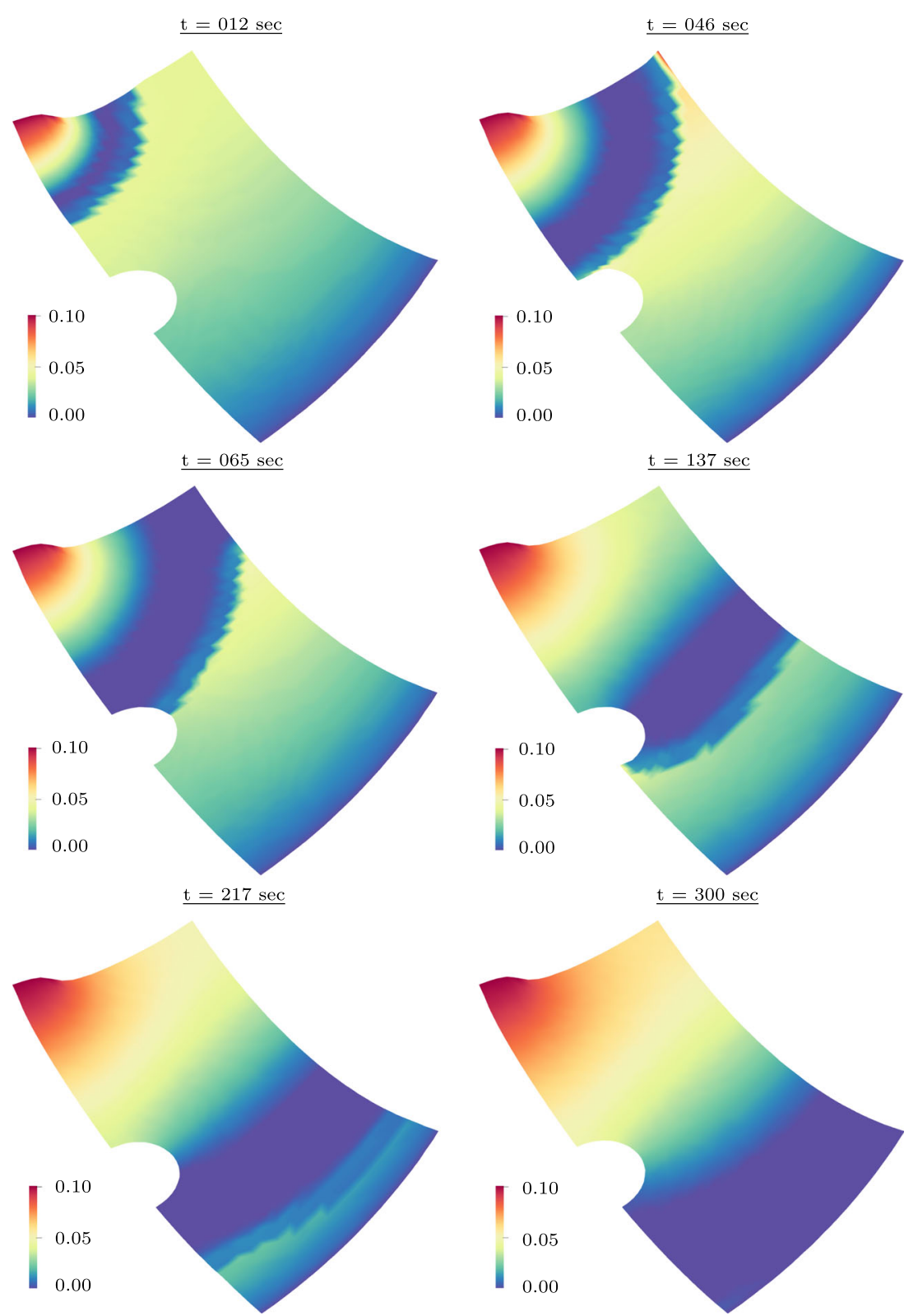


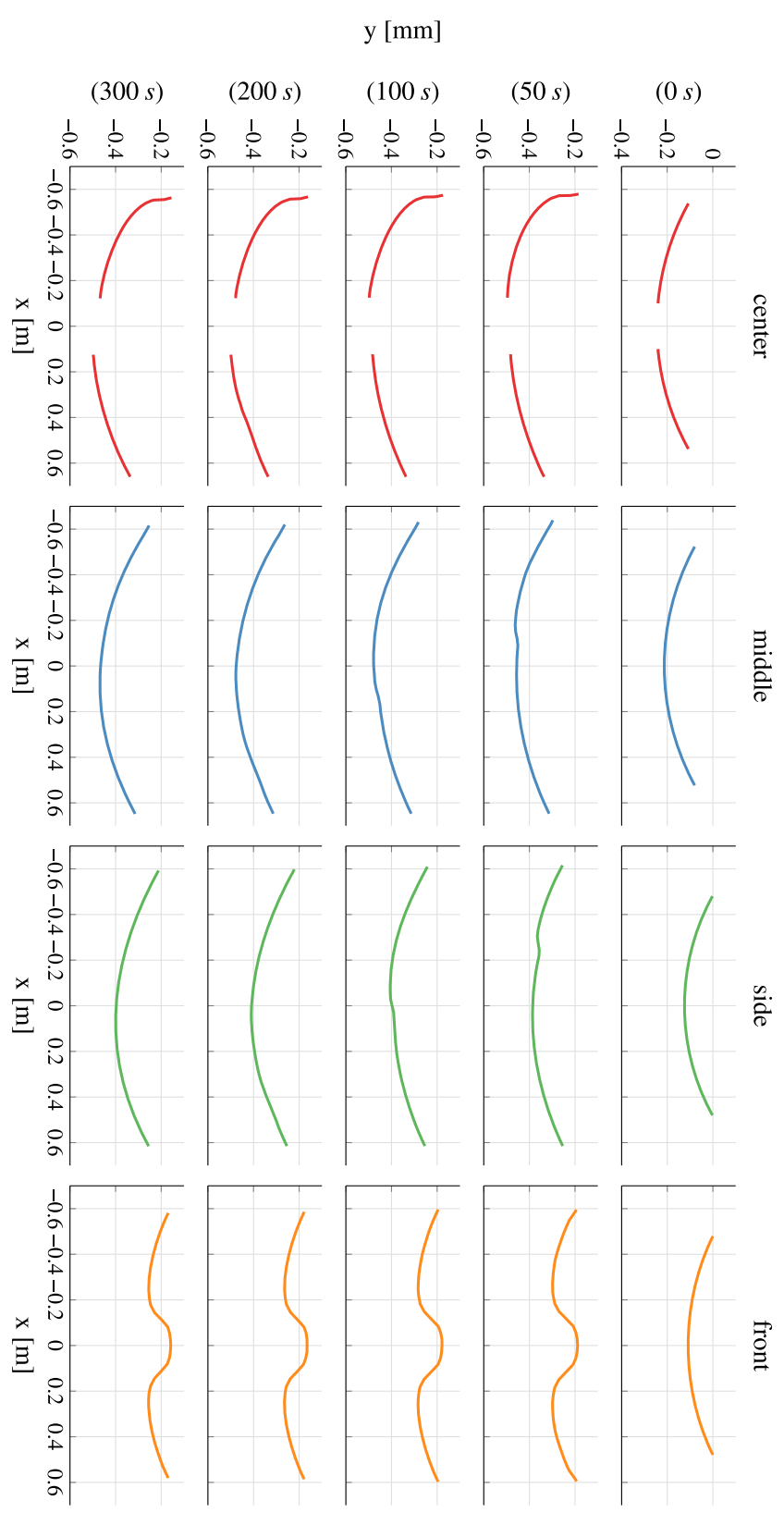

Fig. 10 The deformation of the preform at different locations and times

compressed when the process just begins; after that, the resin moves into and inflates the preform up. From the front section, we can see that the inflation occurs in the vicinity of the inlet. This significant expansion happens immediately after the resin moves in. As the process going, the resin flow moves from the center to sides, which makes the swollen part expanding outwards gradually; and the depth of the swollen part reducing in the meanwhile. What is more, around the inlet, the inflation speed consistently slows down as the pressure approaches the atmospheric pressure.

The center, middle and side section mainly exhibit similar deformation profiles. The longitudinal profiles compress first then expand. The left side parts $(x<0)$ have larger expansion than the right side parts $(x>0)$. At 50, 100 , and 200 seconds, there are local jumps around the flow front regions in the middle and side section. This behavior reflects low pressure bands in Fig. 9. After 200 seconds, the local jumps disappear and profiles turn smoother.

\section{Conclusion}

In this paper, we have presented a novel finite element based model for the LCM process of the thin-walled FRPCM components. The model contributes a shell element in the context of the theory of porous media to model both the preform deformation and the in-plane resin flow. In this fashion, the full 3-D fluid-structure interaction problem is reduced to a $2-\mathrm{D}$ porous media problem, which significantly reduces the number of the degrees of freedom. The model solves for three primary variables: 1 ) the saturation degree $\xi$ and 2) the fluid pressure $p$ are solved from the mass balance relations that describe the homogenized Darcy flow transporting in the porous media; 3) the normal stretch $\lambda$ is then obtained from the explicit formulation Eq. 53, which is derived from the linear momentum balance. The coupled Eqs. 55 and 56 are solved using the StreamlineUpwind/Petrov-Galerkin stabilized finite element method following the staggered approach, as proposed in [15]. The in-plane flow assumption has been justified through the comparison between the 2-D plane and shell models. The convergence studies for the doubly curved specimen shows that the method is converging with respect to the saturation degree for reasonable spatial and temporal discretizations. The model shows the ability to predict different mold filling settings, e.g., multiple resin inlets and different outlet positions. Besides, if the stretch field is prescribed, the same model can also be applied to the RTM process. Thus, this study proposes a simulation tool to optimize the wetout process in the deformable thin-walled fiber preform for large-scale FRPCM components, which can substitute for the costly physical trial and error approaches.

Acknowledgments The authors would like to acknowledge partial support by Swedish Research Council Grant 621-2013-3907 and Chalmers University Area of Advance: Production. The simulations were performed on resources provided by the Swedish National Infrastructure for Computing (SNIC) at Chalmers Center for Computational Science and Engineering (C3SE).

Funding Information Open access funding provided by Chalmers University of Technology.

\section{Compliance with Ethical Standards}

Conflict of interests The authors declare that they have no conflict of interest. 
Open Access This article is distributed under the terms of the Creative Commons Attribution 4.0 International License (http:// creativecommons.org/licenses/by/4.0/), which permits unrestricted use, distribution, and reproduction in any medium, provided you give appropriate credit to the original author(s) and the source, provide a link to the Creative Commons license, and indicate if changes were made.

Nomenclature $\tilde{\xi}$, Global saturation degree; $\boldsymbol{C}$, Right Cauchy-Green deformation tensor; $\boldsymbol{S}$, Effective second Piola-Kirchhoff stress; $\hat{\boldsymbol{S}}$, Total second Piola-Kirchhoff stress; $T$, Absolute temperature; $R$, Ideal gas constant; $m^{g}$, Gas molar mass; $n_{b}$, Capillary pressure constant; $\mu_{l}$, Resin viscosity; $\mu_{g}$, Gas viscosity; $\hat{\boldsymbol{k}}$, Intrinsic permeability of preform; $k_{r l}$, Relative permeability of liquid; $k_{r g}$, Relative permeability of gas; $K^{l}$, Permeability of the preform relating to liquid; $K^{g}$, Permeability of the preform relating to gas; $E$, Young's modulus of fibers; $m$, Packing law exponent; $k^{s}$, Packing law factor; $\psi$, Free energy; $\overline{1}$, In-plane identity tensor; 1, 3-D identity tensor; $K_{\alpha \beta}$, Curvature of the preform; $g^{1}, g^{2}, g^{3}$, Kontra-variant bases of the deformed preform; $\boldsymbol{G}^{1}, \boldsymbol{G}^{2}, \boldsymbol{G}^{3}$, Kontra-variant bases of the undeformed preform; $\boldsymbol{g}_{1}, \boldsymbol{g}_{2}, \boldsymbol{g}_{3}$, Co-variant bases of the deformed preform; $\boldsymbol{G}_{1}, \boldsymbol{G}_{2}, \boldsymbol{G}_{3}$, Co-variant bases of the undeformed preform; $\boldsymbol{E}_{1}, \boldsymbol{E}_{2}, \boldsymbol{E}_{3}$, Inertial Cartesian basis; $\boldsymbol{\Phi}$, Initial position vector; $\theta^{1}, \theta^{2}, \theta^{3}, \theta$, Coordinates; $\lambda$, Stretch; $p^{\text {ent }}$, Entry pressure; $p^{c}$, Capillary pressure; $p^{a}$, Atmosphere pressure; $p^{e}$, Effective pressure of the homogenized fluid; $\hat{p}$, Total pressure of the homogenized fluid; $p$, Homogenized fluid pressure; $\sigma$, Effective stress; $\hat{\boldsymbol{\sigma}}$, Total stress; $\boldsymbol{v}^{d g}$, Darcian velocity of gas; $\boldsymbol{v}^{d l}$, Darcian velocity of liquid; $\boldsymbol{v}^{d f}$, Darcian velocity of the homogenized fluid; $\boldsymbol{v}^{l}$, Velocity of liquid; $\boldsymbol{v}^{g}$, Velocity of gas; $\boldsymbol{v}^{r g}$, Seepage velocity of gas; $\boldsymbol{v}^{r l}$, Seepage velocity of liquid; $\boldsymbol{v}^{r f}$, Seepage velocity of the homogenized fluid; $\boldsymbol{v}^{f}$, Velocity of the homogenized fluid; $\boldsymbol{v}$, Velocity of the solid; $\hat{\rho}$, Density of the homogenized fluid; $\rho^{g}$, Intrinsic density of gas; $\rho^{g}$, Intrinsic density of gas; $\rho^{l}$, Intrinsic density of liquid; $\rho^{f}$, Intrinsic density of homogenized fluid; $\rho^{s}$, Intrinsic density of solid; $M^{l}$, Liquid mass; $M^{f}$, Homogenized fluid mass; $M^{s}$, Solid mass; $\nabla_{\boldsymbol{X}}$, Material gradient operator; $\nabla$, Spatial gradient operator; $J$, Jacobian of $\boldsymbol{F}$; $\boldsymbol{F}$, Deformation gradient; $\varphi$, Deformation mapping; $\overline{\boldsymbol{N}}$, Unit normal of $\Gamma_{0} ; N$, Unit normal of $\Omega_{0} ; \Gamma$, Boundary of $\Omega ; \Gamma_{0}$, Boundary of $\Omega_{0} ; \Omega$, Bottom of the deformed preform; $\Omega_{0}$, Bottom of the undeformed preform; $B$, Region of the deformed preform; $B_{0}$, Region of the undeformed preform; $h$, Thickness of the deformed preform; $h_{0}$, Thickness of the undeformed preform; $\xi$, Saturation degree; $\varphi^{g}$, Volume fraction of the gas; $\varphi^{g}$, Volume fraction of the gas; $\varphi^{l}$, Volume fraction of the liquid; $n^{f}$, Volume fraction of the homogenized fluid phase; $n^{s}$, Volume fraction of solid phase.

\section{References}

1. Advani SG, Hsiao K-T (2012) Manufacturing Techniques for Polymer Matrix Composites (PMCs), Elsevier

2. Chan AW, Hwang S-T (1991) Modeling of the impregnation process during resin transfer molding. Poly Eng Sci 31(15):11491156. https://doi.org/10.1002/pen.760311512

3. Fracchia CA, Castro J, Tucker CL (1989) A finite element/control volume simulation of resin transfer mold filling. In: Proceedings of the American society for composites, fourth technical conference, pp 157-166

4. Bruschke MV, Advani SG (1990) A finite element/control volume approach to mold filling in anisotropic porous media. Polym Compos 11(6):398-405
5. Diallo ML, Gauvin R, Trochu F (1998) Experimental analysis and simulation of flow through multi-layer fiber reinforcements in liquid composite molding. Polym Compos 19(3):246-256. https://doi.org/10.1002/pc.10097

6. Phelan FR (1997) Simulation of the injection process in resin transfer molding. Polym Compos 18(4):460-476. https://doi.org/10.1002/pc.10298

7. Kang MK, Lee WI (1999) A flow-front refinement technique for the numerical simulation of the resin-transfer molding process. Compos Sci Technol 59(11):1663-1674. https://doi.org/10.1016/S0266-3538(99)00029-9

8. Joshi SC, Lam YC, Liu XL (2000) Mass conservation in numerical simulation of resin flow. Compos A: Appl Sci Manuf 31(10):1061-1068. https://doi.org/10.1016/S1359-835X(00)00067-1

9. Um M-K, Lee WI (1991) A study on the mold filling process in resin transfer molding. Polym Eng Sci 31(11):765-771. https://doi.org/10.1002/pen.760311102

10. Soukane S, Trochu F (2006) Application of the level set method to the simulation of resin transfer molding. Compos Sci Technol 66(7):1067-1080. https://doi.org/10.1016/j.comp scitech.2005.03.001

11. Gantois R, Cantarel A, Dusserre G, Félices J-N, Schmidt F (2010) Numerical simulation of resin transfer molding using BEM and level set method. Int $\mathrm{J}$ Mater Form 3(1):635-638. https://doi.org/10.1007/s12289-010-0850-9

12. Binetruy C, Advani SG (2003) Foam core deformation during liquid molding of sandwich structures: Modeling and experimental analysis. Jnl of Sandwich Structures \& Materials 5(4):351-375. https://doi.org/10.1177/109963603027909

13. Comas-Cardona S, Groenenboom P, Binetruy C, Krawczak $\mathrm{P}$, generic mixed A (2005) FE-SPH Method to address hydro-mechanical coupling in liquid composite moulding processes. Compos Part A: Appl Sci Manuf 36(7):1004-1010. https://doi.org/10.1016/j.compositesa.2004.11.009

14. Remacle J, Flaherty J, Shephard M (2003) An adaptive discontinuous galerkin technique with an orthogonal basis applied to compressible flow problems. SIAM Rev 45(1):53-72. https://doi.org/10.1137/S00361445023830

15. Wu D, Larsson $\mathrm{R}$ Homogenized free surface flow in porous media for wet-out processing. International Journal for Numerical Methods in Engineering 1 (17). https://doi.org/10.1002/nme.5812

16. Dammann C, Mahnken R (2019) Simulation of a resin transfer molding process using a phase field approach within the theory of porous media. Compos A: Appl Sci Manuf 120:147-160. https://doi.org/10.1016/j.compositesa.2019.02.022

17. Niaki SA, Forghani A, Vaziri R, Poursartip A (2018) A three-phase integrated flow-stress model for processing of composites. Mech Mater 117:152-164. https://doi.org/10.1016/j. mechmat.2017.10.012

18. Li M, Tucker CL (2002) Modeling and simulation of two-dimensional consolidation for thermoset matrix composites. Compos A: Appl Sci Manuf 33(6):877-892. https://doi.org/10.1016/S1359-835X(02)00017-9

19. Wysocki M, Larsson R, Toll S (2005) Hydrostatic consolidation of commingled fibre composites. Compos Sci Technol 65(10):15071519. https://doi.org/10.1016/j.compscitech.2005.01.002

20. Wysocki M, Asp LE, Toll S, Larsson R (2009) Two phase continuum modelling of composites consolidation. Plast Rubber Compos 38(2-4):93-97. https://doi.org/10.1179/174328909X387856

21. Wysocki M, Toll S, Larsson R, Asp LE (2010) Anisotropic and tension-compression asymmetric model for composites consolidation. Compos A: Appl Sci Manuf 41(2):284-294. https://doi.org/10.1016/j.compositesa.2009.10.016 
22. Larsson R, Rouhi M, Wysocki M (2012) Free surface flow and preform deformation in composites manufacturing based on porous media theory. Eur J Mech - A Solid 31(1):1-12. https://doi.org/10.1016/j.euromechsol.2011.06.015

23. Rouhi MS, Wysocki M, Larsson R (2013) Modeling of coupled dual-scale flow-deformation processes in composites manufacturing. Compos A: Appl Sci Manuf 46:108-116. https://doi.org/10.1016/j.compositesa.2012.11.002

24. Rouhi MS, Wysocki M, Larsson R (2015) Experimental assessment of dual-scale resin flow-deformation in composites processing. Compos A: Appl Sci Manuf 76:215-223. https://doi.org/10.1016/j.compositesa.2015.05.034

25. Rouhi MS, Wysocki M, Larsson R (2016) Holistic modeling of composites manufacturing using poromechanics. Advanced Manufacturing: Polymer \& Composites Science 2(1):14-26. https://doi.org/10.1080/20550340.2016.1141457

26. Blais M, Moulin N, Liotier P.-J., Drapier S (2017) Resin infusionbased processes simulation : coupled stokes-darcy flows in orthotropic preforms undergoing finite strain. Int $\mathrm{J}$ Mater Form 10(1):43-54. https://doi.org/10.1007/s12289-015-1259-2

27. Andriamananjara K, Moulin N, Bruchon J, Liotier P-J, Drapier S Numerical modeling of local capillary effects in porous media as a pressure discontinuity acting on the interface of a transient bi-fluid flow. Int $\mathrm{J}$ Mater Form. https://doi.org/10.1007/s12289-018-1442-3

28. Mathur R, Advani SG, Gillespie JrJ., Fink B (2000) A closed form solution for flow during the vacuum assisted resin transfer molding process. J Manuf Sci Eng 122:463-475

29. Correia NC, Robitaille F, Long AC, Rudd CD, Simacek P, Advani SG (2004) Use of resin transfer molding simulation to predict flow, saturation, and compaction in the VARTM process. J Fluids Eng 126(2):210-215

30. Correia NC, Robitaille F, Long AC, Rudd CD, Šimáček P, Advani SG (2005) Analysis of the vacuum infusion moulding process: I. Analytical formulation. Compos A: Appl Sci Manuf 36(12):16451656. https://doi.org/10.1016/j.compositesa.2005.03.019

31. Li J, Zhang C, Liang R, Wang B, Walsh S (2008) Modeling and analysis of thickness gradient and variations in vacuum-assisted resin transfer molding process. Polym Compos 29(5):473-482. https://doi.org/10.1002/pc.20439
32. LeBel F, Fanaei AE, Ruiz E, Trochu F (2014) Prediction of optimal flow front velocity to minimize void formation in dual scale fibrous reinforcements. Int J Mater Form 7(1):93-116. https://doi.org/10.1007/s12289-012-1111-x

33. Fracassi FT, Donadon MV (2018) Simulation of vacuum assisted resin transfer molding process through dynamic system analysis. J Compos Mater 52(27):3759-3771. https://doi.org/10.1177/0021998318770000

34. Hsiao K.-T., Devillard M, Advani SG (2004) Simulation based flow distribution network optimization for vacuum assisted resin transfer moulding process. Modelling Simul Mater Sci Eng 12(3):S175. https://doi.org/10.1088/0965-0393/12/3/S08

35. Toll S (1998) Packing mechanics of fiber reinforcements. Polym Eng Sci 59(8):133-150

36. Larsson R, Wysocki M, Toll S (2004) Process-modeling of composites using two-phase porous media theory. Europ J Mech A Solid 23(1):15-36. https://doi.org/10.1016/j.euromechsol.2003. 09.004

37. Betsch P, Stein E (1996) A nonlinear extensible 4-node shell element based on continuum theory and assumed strain interpolations. J Nonlinear Sci 6:169-199

38. Javili A, McBride A, Steinmann P (2013) Numerical modelling of thermomechanical solids with highly conductive energetic interfaces. Int J Numer Meth Eng 263:1-11

39. Gebart B (1992) Permeability of unidirectional reinforcements for RTM. J Compos Mater 26:1100

40. Burdine N (1953) Relative permeability calculations from pore size distribution data. J Pet Technol 5(03):71-78. https://doi.org/10.2118/225-G

41. Brooks RH, Corey AT (1964) Hydraulic properties of porous media and their relation to drainage design. Transactions of the ASAE 7(1):0026-0028. https://doi.org/10.13031/2013.40684

Publisher's note Springer Nature remains neutral with regard to jurisdictional claims in published maps and institutional affiliations. 